\title{
Empowerment perception and health-related quality of life among schizophrenic patients
}

\author{
Hsiao-Tan Chiu ${ }^{1}$, Hui-Man Huang ${ }^{* 2}$ \\ ${ }^{1}$ Department of Nursing, Jianan Psychiatric Center, Ministry of Health Welfare, Taiwan (R.O.C.) \\ ${ }^{2}$ Department of Nursing, National Quemoy University, Taiwan (R.O.C.)
}

Received: November 15, 2020

DOI: $10.5430 / \mathrm{cns} . v 9 \mathrm{n} 1 \mathrm{p} 45$
Accepted: January 5, 2021

Online Published: January 27, 2021

\begin{abstract}
Objective: The study aims to explore the perception of empowerment, HRQoL and their relationship in schizophrenia patients in rehabilitation ward.

Methods: Cross-sectional correlational design and convenience sampling were used to recruit 102 subjects from rehabilitation wards of a psychiatric hospital in southern Taiwan. Three instruments were used: the Brief Psychiatric Rating Scale, the Empowerment Scale (subscales: self-esteem and self-efficacy, power-powerlessness, righteous anger, and community autonomy), and the Taiwanese version of the World Health Organization Quality of Life Questionnaire (WHOQOL-BREF) with four domains (physical health, psychological, social relationship, and environment).

Results: (1) Overall empowerment perception and overall HRQoL were at a moderate and above level in patients with schizophrenia in rehabilitation ward. (2) Older patients having a lower empowerment perception with power-powerlessness than youngers; the more severe the psychiatric symptoms, the lower the righteous anger. (3) Male patients scored higher than female patients on overall HRQoL and "environment" domain. The duration of mental illness also influenced at "environment" domain, 6-10 years group scored higher than $\leq 5$ years group. (4) Actively participate in rehabilitation activities correlated positively; psychiatric symptoms correlated negatively with overall HRQoL and with each individual domain. (5) Empowerment perception correlated positively with overall HRQoL and with each individual domain.

Conclusions: Mental health workers should assist chronic schizophrenic patients in coping with their psychiatric symptoms, allow patients to express negative emotions, and provide opportunities for patients to make their own decisions and exercise their own rights.
\end{abstract}

Key Words: Empowerment, Health-related quality of life, Schizophrenia, Rehabilitation ward

\section{INTRODUCTION}

Schizophrenia is an early-onset and chronic psychiatric disorder characterized by its deterioration course. Patients who suffer from schizophrenia have to undergo long-term medical treatment at psychiatric acute or rehabilitation wards. After developing schizophrenia, a patient has to take medication regularly to stabilize his or her condition and cope with the psychological and social impact of the disease. Beyond remission of psychiatric symptoms, recovery-oriented mental health professionals support the patients with schizophrenia to maintain individual rights to self-determination, remain active in their community, and full inclusion in community life, regardless of disability status. During the recovery process, schizophrenic patients who receive affirmation for their

\footnotetext{
*Correspondence: Hui-Man Huang; Email: x2156@nqu.edu.tw; Address: Department of Nursing, National Quemoy University, No. 1, University Rd., Jinning Township, Kinmen County, 89250, Taiwan (R.O.C.).
} 
work, economic, and individual abilities, experience social acceptance and self-empowerment. These factors help them to perform the limited roles they acquire. ${ }^{[1]}$

Lower quality of life (QoL) has been associated with schizophrenia patients. ${ }^{[2]}$ There has been a shift in mental health services with treatment that is focused on reducing symptoms to a more holistic approach which considers QoL. ${ }^{[3,4]}$ Tseng, Chiou, Yen et al. ${ }^{[5]}$ pointed out that research into the health-related quality of life (HRQoL) of schizophrenic patients allows health professionals to develop a stronger understanding of the quality of life of such patients. This places them in a substantially better position to create future rehabilitation programs. Shih ${ }^{[6]}$ emphasized that the empowerment perception has a significant impact on the HRQoL of schizophrenic patients. This indicates that it is worthwhile to investigate the feeling of empowerment and HRQoL with respect to schizophrenic patients.

\section{LiterATURE REVIEW}

\subsection{Characteristics of schizophrenic patients in rehabili- tation wards}

The symptoms of schizophrenia are associated with, cognitive, perceptual, and relational dysfunctions that cause patients to make erroneous judgments about their external environment, or even to isolate themselves socially, which in turn affects their academic and occupational abilities and their quality of life. ${ }^{[7,8]}$ Chronic schizophrenic patients who undergo rehabilitation in hospitals mostly endure longer periods of affliction and moderate mental symptoms; their conditions have yet to stabilize and they still require long-term care and rehabilitation. In addition to receiving medication, schizophrenic patients also undergo continuous rehabilitation in a rehabilitation ward to stabilize their symptoms and fully utilize their existing functions. The therapeutic activities in a rehabilitation ward include disease and drug education, lessons covering effective coping behaviors, occupational rehabilitation training, and the provision of social resources and information. The objective of these activities is to improve the self-care ability and daily living functions of the rehabilitation patients, such that they will be able to re-integrate into the community. ${ }^{[9]}$

\subsection{Schizophrenic patients' empowerment perception and related influencing factors}

$\mathrm{Wu}$, Hong, and Chen ${ }^{[10]}$ proposed that empowerment is a process through which an individual acquires self-control, and the individual establishes this process through sharing, cooperation, participation with respect to decision-making, and autonomous activities. The basic attributes of empowerment are: having decision making power, assertiveness, a feeling that one can make a difference, learning about and expressing anger, not feeling alone, feeling part of a group, understanding that a person has rights, growth and change that are never-ending and self-initiated, increasing one's positive self-image. ${ }^{[11]}$ Empowerment plays an important role in the rehabilitation process for psychiatric patients. ${ }^{[6,11,12]}$ Empowerment also indicates a sense of personal competence which is considered an essential vital of fair outcome in schizophrenia. ${ }^{[13]}$ Symptom severity is a factor that influences empowerment in psychiatric patients. Shih ${ }^{[6]}$ examined the empowerment perception of 190 patients with chronic schizophrenia from a community psychiatric rehabilitation institution, and Hansson and Björkmn ${ }^{[14]}$ explored the empowerment perception of 97 psychiatric patients; both studies revealed a negative correlation between mental symptoms and empowerment perception. In addition, Vauth, Kleim, Wirtz et al. ${ }^{[15]}$ investigated 172 schizophrenic outpatients and the results suggested that their depression symptoms were exacerbated when they felt less empowered. Furthermore, active participation in community rehabilitation activities can also help increase the empowerment perception of schizophrenic patients. ${ }^{[6,11,15]}$

\subsection{Schizophrenic patients' HRQoL and the related in- fluencing factors}

HRQoL representing a patient's subjective health state, reflects goals of patient-center care and is therefore attracting growing attention in the medical community. ${ }^{[16]}$ Tseng, Chiou, Yen et al. ${ }^{[5]}$ indicated that the maintenance of HRQoL is a continuous adaptation process that can help patients cope with stress and respond to the surrounding environment. In addition, health-related quality of life is acknowledged as an indicator of psychiatric rehabilitation outcomes. ${ }^{[2,16]}$ Healthrelated quality-of-life measures could help psychiatric professionals obtain a holistic view of the quality of life of patients with schizophrenia. ${ }^{[3]}$ Accordingly, the assessment of the HRQoL of schizophrenic patients is a crucial clinical and research topic. Personal and illness characteristics including gender, duration of illness, symptom severity, and participation in rehabilitation activities have been associated with quality of life in patients with schizophrenia. Tseng[17] looked at the HRQoL of schizophrenic patients in the community and found that the male schizophrenic patients had a higher perceived quality of life compared to female patients. Shih $^{[6]}$ indicated that schizophrenic patients in the community who had lived with the disease over a longer period of time experienced a poorer HRQoL. Both Tseng ${ }^{[17]}$ and Chou, Shih, Chang et al. ${ }^{[18]}$ emphasized the presence of a significant negative correlation between symptom severity and overall quality of life. Shih ${ }^{[6]}$ found that, among the schizophrenic patients who participated in the mental health 
rehabilitation programs implemented by their communities, $85 \%$ of them had experienced an improved quality of life; additionally, Chou, Shih, Chang et al. ${ }^{[18]}$ echoed this finding, as well.

\subsection{Relationship between empowerment perception and HRQoL among schizophrenic patients}

There is a link between stronger empowerment perception and increased quality of life. ${ }^{[11,18]}$ In a study by Corrigan, Faber, Rashid et al. ${ }^{[12]}$ explored the empowerment perception of 35 hospitalized psychiatric patients and found that self-direction was significantly correlated to their quality of life. Hansson and Björkman ${ }^{[14]}$ pointed out that a positive correlation existed between the self-esteem and activism aspects of an individual's empowerment perception and his or her quality of life. Both Vauth, Kleim, Wirtz et al. ${ }^{[15]}$ and Chou, Shih, Chang et al. ${ }^{[18]}$ indicated that a decreased level of empowerment leads to a decline in the quality of life of schizophrenic patients. Additionally, an individual's HRQoL is also positively correlated with his or her empowerment perception. ${ }^{[19,20]} \mathrm{Shih}^{[6]}$ stressed that there is a significant positive correlation between empowerment perception and HRQoL. Chou, Shih, Chang et al. ${ }^{[18]}$ also pointed out that psychiatric rehabilitative programs indirectly influence a patient's quality of life through their impact on his or her empowerment perception. In order to raise a patient's quality of life through his or her participation in rehabilitation activities, it is therefore necessary to address these issues.

In summary, mental health professionals should examine ways to increase the empowerment perception and HRQoL of schizophrenic patients. However, past studies on the quality of life of psychiatric patients have mostly focused on community or OPD psychiatric patients. ${ }^{[5,18,21]}$ Although Shih's ${ }^{[6]}$ findings support the existence of a link between empowerment perception and HRQoL, no studies have been conducted as yet on schizophrenic patients in rehabilitation wards who suffer from long-term residual symptoms. In light of these considerations, the aims of this study were to: (1) understand the empowerment perception and HRQoL of schizophrenic patients in rehabilitation wards, as well as the influencing factors for these two variables; and (2) investigate the relationship between the empowerment perception and HRQoL of schizophrenic patients in rehabilitation wards. It is hoped that the results of this study will contribute to efforts by mental health professionals to increase the empowerment perception and HRQoL of schizophrenic patients in rehabilitation wards.

\section{MethodS}

In this correlational study, a cross-sectional design and three structured questionnaires were used to collect data from

Published by Sciedu Press schizophrenic patients in rehabilitation wards.

\subsection{Participants}

The study used convenience sampling to select 102 schizophrenic patients from the rehabilitation ward of a psychiatric hospital in southern Taiwan. The selection criteria were as follows: (1) the patient was diagnosed with schizophrenia (fulfilled criteria of DSM-5) by a psychiatrist; (2) the patient had been hospitalized in the rehabilitation ward for more than one month; (3) the patient was able to fill out the questionnaires or respond to interview questions; and (4) the patient had signed the participant consent form. The exclusion criteria were as follows: (1) the patient was diagnosed with mental retardation; (2) the patient was suffering from cognitive dysfunction as a result of a brain injury or dementia; and (3) the patient displayed prominent residual symptoms.

\subsection{Data collection process and ethical considerations}

The study was reviewed and approved by an institutional review board (Jianan 15-024), and its data was then collected from six rehabilitation wards from November 1 to December 31, 2015. To protect the rights and interests of the participants, the researchers visited the wards in person to explain the research process to the participants. With each participant, the researcher first confirmed whether he or she could answer questions correctly. Next, the patient was requested to complete an empowerment scale form and a HRQoL scale form. While the participants completed the questionnaires, the researcher observed and later interviewed the patient and completed a short mental symptoms scale form. The data for three questionnaire items - the length of time since the schizophrenia diagnosis, the level of participation in rehabilitation activities, and the frequency of out-of-hospital adaptation therapy-were obtained from the patients' medical records.

\subsection{Instruments}

\subsubsection{Patient demographics and disease characteristics questionnaire}

(1) Patient demographics: age, gender, education level, religious affiliation, and marital status.

(2) Disease characteristics: length of time since schizophrenia diagnosis, symptom severity, frequency of out-of-hospital adaptation therapy, and level of participation in rehabilitation activities. The Brief Psychiatric Rating Scale (BPRS) was used to assess the symptom severity of the patients. The BPRS form consists of 18 questions, uses a seven-point Likert scale, and allows for a total score of 18-126 points. The higher a patient's score was, the more severe his or her symptoms were. In a study by Chang, Hwu, and Wei, ${ }^{[22]}$ the BPRS 
scale was used and a reliability level of .8 was achieved. The frequency of out-of-hospital adaptation therapy refers to the mean number of times, in a quarter, that a patient had engaged in out-of-hospital community interactions (including outings, overnight stays, and community activities) during his or her hospitalization. The level of participation in rehabilitation activities was determined by observing the patients' participation in mental health rehabilitation activities and sorting them into the following five categories: did not need encouragement to participate; able to participate after being encouraged once; able to participate after being encouraged twice or thrice; able to participate after being encouraged multiple times; did not participate.

\subsubsection{Empowerment scale}

The empowerment scale developed by Rogers, Chamberlin, Ellison et al. ${ }^{[11]}$ was utilized to understand the empowerment perception of patients. This scale contains 28 questions covering five domains: self-esteem (9 items), powerpowerlessness ( 7 items), community activism and autonomy (5 items), control over the future (4 items), and righteous anger (3 items). A four-point Likert scale was used, with 1 point representing "strongly agree" and 4 points representing "strongly disagree." Of the items, Items 1, 3, 5, 6, 9, 11-15, 18-20, and 24-28 are reverse-scored items. The score range is $28-112$, with a higher total score representing stronger empowerment perception. Before the study started, the Chinese version of the empowerment scale was reviewed by a bilingual nursing expert, who revised the Chinese phrasing for the second dimension and the items, and by two psychiatric clinical nursing experts, who checked the appropriateness of each question. The expert validity for the scale was .91. Chen, Liu, Hsieh et al. ${ }^{[23]}$ applied the scale in a study involving 178 psychiatric patients and reported a Cronbach's $\alpha$ value of .87; after the study was conducted, the scale's Cronbach's $\alpha$ value was found to be .7. Therefore, the Empowerment Scale appears to be a suitable instrument to capture the dimension of empowerment in Taiwan's mental health settings.

\subsubsection{The World Health Organization's Quality of Life- Brief (WHOQOL-BREF)}

This study used the World Health Organization's Quality of Life-Brief (WHOQOL-BREF) Taiwan version questionnaire that was adapted by Yao. ${ }^{[24]}$ This questionnaire was based on the World Health Organization Quality of Life (WHOQOL) questionnaire, which was published by the WHOQOL Group. This scale questionnaire consists of 28 items, including 26 questions that cover overall quality of life (four domains, 24 questions) and general HRQoL (two questions), and two additional localized questions: "Do you feel respected?" and "Do you usually get to eat what you want?". The four domains for overall quality of life are: (1) physical health domain (seven questions); (2) mental domain (six questions); (3) social relationship domain (four questions); and (4) environment domain (nine questions). A five-point Likert scale is applied, with 1 point representing "not important" and 5 points representing "very important." Of the questions, questions 3, 4, and 26 are reverse-scored questions. The score range for each domain is 4-20 points, and the total score range for the 26 questions is $16-80$ points. A higher score for a question indicates a better quality of life with respect to this question. Yao ${ }^{[25]}$ pointed out that the WHOQOL-BREF scale provides a high level of reliability and validity, with the overall scale and four domains having a Cronbach's $\alpha$ of .91 and .70-.77. With regard to construct validity, the four domains can explain $73 \%$ of the variance. After the study was implemented, the scale's Cronbach's $\alpha$ was found to be .91 , indicating that this scale had an acceptable level of reliability and validity.

\subsection{Data analysis}

The data were analyzed using SPSS Version 20.0 with the alpha level set at .05 . The statistical analysis covered frequency distribution, percentages, mean values, and standard deviation and performed t-test, one-way ANOVA, and Pearson product-moment correlation tests.

\section{RESUlts}

\subsection{Patient demographics and disease characteristics,} empowerment perception, and HRQoL

Table 1 breaks down the patients' demographics and disease characteristics. The patients' mean age was 44.02 \pm 9.78 , and most of the patients were male $(72.5 \%)$, senior/vocational high school-educated $(40.2 \%)$, religious (80.3\%), and unmarried (87.2\%). Most of the patients have lived with the disease for 21 years or more (41.1\%) and had mild symptom severity $(27.7 \pm 6.4)$, which indicated that their conditions were mostly stable. With respect to the frequency of out-of-hospital adaptation therapy, most of the patients participated in out-of-hospital community interactions 1-3 times per quarter (36.2\%). As for their level of participation in rehabilitation activities, most patients did not need encouragement to participate in these activities (36.2\%). The patients' overall empowerment perception was moderately strong (mean score of $80.65 \pm 21.56$ ), and with respect to the domains, the highest score was generated by the community activism and autonomy domain (3.22), whereas the lowest score was generated by the power-powerlessness domain (2.42). The patients' HRQoL was determined to be moderately high (mean score of $51.43 \pm 15.72$ ), and the score ranking of its four domains (in descending order) is as follows: social relationship domain, environmental domain, physiological domain, and psychological domain. 
Table 1. Patient demographics, disease characteristics, and symptom severity $(\mathrm{N}=102)$

\begin{tabular}{|c|c|c|c|}
\hline Variable & n $(\%)$ & Variable & $\mathrm{n}(\%) / \mathrm{M} \pm S D$ \\
\hline Age $(M \pm S D)$ & $44.02 \pm 9.78$ & \multicolumn{2}{|l|}{ Number of years lived with disease } \\
\hline $21-30$ & $11(10.8)$ & $\leq 5$ & $10(9.8)$ \\
\hline $31-40$ & $24(23.5)$ & $\overline{6}-10$ & $17(16.6)$ \\
\hline $41-50$ & $37(36.3)$ & $11-20$ & $33(32.3)$ \\
\hline $51-60$ & $27(26.5)$ & $\geq 21$ & $42(41.1)$ \\
\hline $61-70$ & $3(2.9)$ & \multicolumn{2}{|l|}{ Symptom severity } \\
\hline Gender & & Overall symptoms score range & $18-49$ \\
\hline Male & $74(72.5)$ & \multirow[t]{2}{*}{ Overall symptoms mean $(\mathrm{M} \pm S D)$} & $27.7 \pm 6.4$ \\
\hline Female & $28(27.5)$ & & \\
\hline Education level & & \multicolumn{2}{|c|}{ Frequency of out-of-hospital adaptation therapy (number of times/season) } \\
\hline Elementary school & $9(8.8)$ & 0 & $12(11.7)$ \\
\hline Junior high school & $32(31.4)$ & $1-3$ & $37(36.2)$ \\
\hline Senior high school/vocation school & $41(40.2)$ & $4-6$ & $22(21.5)$ \\
\hline University/college or higher & $20(19.6)$ & $7-9$ & $31(30.3)$ \\
\hline Religious affiliation & & \multicolumn{2}{|l|}{ Level of participation in rehabilitation activities } \\
\hline No & $20(19.6)$ & Did not need encouragement to participate & $37(36.2)$ \\
\hline Yes & $82(80.4)$ & Able to participate after being encouraged once & $36(35.2)$ \\
\hline \multicolumn{4}{|l|}{ Marital status } \\
\hline Unmarried & $89(87.2)$ & \multirow{2}{*}{$\begin{array}{l}\text { Able to participate after being encouraged multiple times } \\
\text { Did not participate }\end{array}$} & \multirow{2}{*}{$\begin{array}{l}7(6.8) \\
0(0.0)\end{array}$} \\
\hline Divorced/widowed & $8(7.9)$ & & \\
\hline
\end{tabular}

4.2 Relationship of patient demographics with empowerment perception and HRQoL

Table 2 shows that older patients scored lower $(r=-0.200$, $p<.05)$ on the power-powerlessness domain of the empowerment perception scale, whereas patients with milder symptom severity scored higher $(r=-0.198, p<.05)$ on the righteous anger domain of the empowerment perception scale. Table 3 demonstrates that symptom severity is significantly and negatively correlated with overall HRQoL $(r=-0.292$, $p<.05)$ and all its domains, namely, the physiological domain $(r=-0.309, p<.01)$, psychological domain $(r=-0.239$, $p<.05)$, social relationship domain $(r=-0.284, p<.01)$, and environmental domain $(r=-0.216, p<.01)$. This meant that patients with milder symptom severity had a higher HRQoL. The level of participation in rehabilitation activities was found to be significantly and positively correlated with overall HRQoL $(r=0.372, p<.001)$ and all its domains, namely, the physiological domain $(r=0.433, p<.001)$, psychological domain $(r=0.455, p<.001)$, social relationship domain $(r=0.376, p<.001)$, and environmental domain $(r=$
$0.470, p<.001)$. This meant that patients who did not need encouragement to participate in activities enjoyed a higher HRQoL.

Table 4 shows that the male patients reported a higher level of HRQoL satisfaction than the female patients with respect to overall HRQoL $(p<.05)$ and the environmental domain $(p<$ $.05)$. Compared with patients who had lived with the disease for 5 years or fewer, those who had lived with the disease for 6-10 years reported higher scores for the environmental domain $(p<.05)$.

\subsection{Relationship between patients' empowerment per- ception and HRQoL}

Table 5 shows that the patients' overall empowerment perception were significantly and positively correlated with overall HRQoL $(r=0.570, p<.001)$ and its four domains, namely, the physiological domain $(r=0.527, p<.001)$, psychological domain $(r=0.605, p<.001)$, social relationship domain $(r=$ $0.436, p<.001)$, and environmental domain $(r=0.419, p<$ $.001)$.

Table 2. Correlation analysis of patient demographics, disease characteristics and empowerment perception $(\mathrm{N}=102)$

\begin{tabular}{|c|c|c|c|c|c|c|}
\hline Variables & $\begin{array}{l}\text { Overall empowerment } \\
\text { perception }\end{array}$ & Self-esteem & $\begin{array}{l}\text { Power- } \\
\text { powerlessness }\end{array}$ & $\begin{array}{l}\text { Community activism } \\
\text { and autonomy }\end{array}$ & $\begin{array}{l}\text { Control over the } \\
\text { future }\end{array}$ & $\begin{array}{l}\text { Righteous } \\
\text { anger }\end{array}$ \\
\hline Age & -.058 & .052 & $-.200^{*}$ & -.043 & .096 & .041 \\
\hline Symptom-severity & -.056 & -.025 & -.001 & -.079 & -.102 & $-.198^{*}$ \\
\hline
\end{tabular}

Note. ${ }^{*} p<.05$

Table 3. Correlation analysis of patient demographics, disease characteristics and HRQoL $(\mathrm{N}=102)$

\begin{tabular}{llllll}
\hline Variables & Overall HRQoL & Physiological & Psychological & Social relationship & Environmental \\
\hline Symptom severity & $-.292^{*}$ & $-.309^{* *}$ & $-.239^{*}$ & $-.284^{* *}$ & $-.216^{* *}$ \\
Level of participation in rehabilitation activities & $.372^{* * *}$ & $.433^{* * *}$ & $.455^{* * *}$ & $.376^{* * *}$ & $.470^{* * *}$ \\
\hline
\end{tabular}

Note. ${ }^{*} p<.05 ;{ }^{* *} p<.01 ;{ }^{* * *} p<.001$ 
Table 4. Analysis of difference in HRQoL based on patient demographics, disease characteristics $(\mathrm{N}=102)$

\begin{tabular}{|c|c|c|c|c|c|c|c|c|c|c|c|}
\hline \multirow{2}{*}{ Variables } & \multirow{2}{*}{$\mathbf{n}$} & \multicolumn{2}{|c|}{ Overall HRQoL } & \multicolumn{2}{|c|}{ Physiological } & \multicolumn{2}{|c|}{ Psychological } & \multicolumn{2}{|c|}{ Social relationship } & \multicolumn{2}{|c|}{ Environmental } \\
\hline & & $M \pm S D$ & $\mathbf{t} / \mathbf{F}$ & $M \pm S D$ & $\mathbf{t} / \mathbf{F}$ & $M \pm S D$ & $\mathbf{t} / \mathbf{F}$ & $M \pm S D$ & $\mathbf{t} / \mathbf{F}$ & $M \pm S D$ & $\mathbf{t} / \mathbf{F}$ \\
\hline Gender & & & $2.15^{*}$ & & 1.57 & & 1.85 & & 1.67 & & $2.21^{*}$ \\
\hline Male & 74 & $3.29 \pm 0.53$ & & $3.27 \pm 0.56$ & & $3.20 \pm 0.65$ & & $3.34 \pm 0.68$ & & $3.33 \pm 0.58$ & \\
\hline Female & 28 & $3.02 \pm 0.52$ & & $3.07 \pm 0.47$ & & $2.91 \pm 0.61$ & & $3.07 \pm 0.75$ & & $3.02 \pm 0.64$ & \\
\hline \multicolumn{3}{|c|}{ Number of years lived with disease } & 1.34 & & 2.45 & & 1.01 & & 1.26 & & $2.91^{*}$ \\
\hline a. $\leq 5$ years & 10 & & & $3.04 \pm 0.55$ & & $2.80 \pm 0.72$ & & $2.92 \pm 0.64$ & & $2.83 \pm 0.67$ & \\
\hline b. $6-10$ years & 17 & & & $3.24 \pm 0.74$ & & $3.34 \pm 0.79$ & & $3.44 \pm 0.69$ & & $3.52 \pm 0.70$ & \\
\hline c. $11-20$ years & 33 & & & $3.19 \pm 0.44$ & & $3.06 \pm 0.53$ & & $3.21 \pm 0.70$ & & $3.21 \pm 0.53$ & \\
\hline d. $\geq 21$ years & 42 & & & $3.27 \pm 0.52$ & & $3.17 \pm 0.64$ & & $3.32 \pm 0.72$ & & $3.25 \pm 0.57$ & \\
\hline Scheffe's test & & & & & & & & & & $b>a$ & \\
\hline
\end{tabular}

Note. ${ }^{*} p<.05$

Table 5. Correlation analysis of empowerment perception and HRQoL

\begin{tabular}{llllll}
\hline Variables & Overall HRQoL & Physiological domain & Psychological domain & Social relationship domain & Environmental domain \\
\hline $\begin{array}{l}\text { Overall empowerment } \\
\text { perception }\end{array}$ & $.570^{* * *}$ & $.527^{* * *}$ & $.605^{* * *}$ & $.436^{* * *}$ & $.419^{* * *}$ \\
\hline Note. ${ }^{* * *} p<.001$ & & & &
\end{tabular}

\section{DISCUSSION}

\subsection{Schizophrenic patients' empowerment perception}

The results showed that schizophrenic patients in the rehabilitation ward had a moderately high empowerment perception. For the four domains of empowerment perception, the community activism and autonomy domain had the highest score (3.22), and the power-powerlessness domain had the lowest score. These findings and the ranking of the domains were similar to those obtained by Shih, ${ }^{[6]}$ who investigated the empowerment perception of chronic schizophrenic patients from a community psychiatric rehabilitation institution. However, the community activism and autonomy domain score in this study was higher than Shih's ${ }^{[6]}$ by 0.27 points, which could be explained to the fact that the patients of this study often participated in mental health rehabilitation activities and engaged in close interactions with the community $(51.8 \%$ of the patients participated in out-of-hospital adaptation therapy more than five times every quarter). This showed that chronic schizophrenic patients reported less of the power-powerlessness domain of empowerment perception even though they lived in different environments. Furthermore, this study also showed that older adults experienced less power-powerlessness of empowerment perception, which differed from the results obtained by Shih. ${ }^{[6]}$ This may be because these older adults have experienced more constraints to the exercise of power as they have been affected by the disease for a longer period of time, thus resulting in a lower level of power-powerlessness. Symptom severity was found to be negatively correlated to the righteous anger domain of empowerment perception. This finding was consistent with those obtained by Hansson and Björkman ${ }^{[14]}$ and Vauth, Kleim, Wirtz et al., ${ }^{[15]}$ who uncovered a negative correlation between symptom severity and empowerment perception. This suggested that patients with milder symptom severity were better able to express negative emotions linked to the righteous anger domain.

\subsection{HRQoL in schizophrenic patients}

The overall HRQoL of schizophrenic patients in rehabilitation wards was moderately high (mean value of 51.43 \pm 15.72 ), which was consistent with the results obtained by Shih. ${ }^{[6]}$ However, of the four domains, the social relationship domain had the highest score, which differed from recent studies, in which the highest scores were generated by the physiological. ${ }^{[5,26,27]}$ The participants reported a higher level of satisfaction in the social relationship domain. This could be linked to the fact that the rehabilitation wards investigated in this study were promoting job training at the hospital and mutual cooperation among patients in the same ward. Furthermore, efforts were also made to cultivate an accepting attitude among the medical staff toward the patients. The psychological domain had the lowest score in HRQoL, indicating that the long-term effects of the disease on the patients have led to the development of feelings of inferiority and a vulnerable self-esteem. Thus, many patients suffered from low self-esteem and rated their abilities more lowly, ${ }^{[28]}$ and as a result, reported a lower level of satisfaction for the psychological domain of HRQoL. The results showed that, compared with female patients, male patients enjoyed a higher overall HRQoL and environmental domain HRQoL, which was consistent with the results obtained by Tseng. ${ }^{[17]}$ However, in a study by Lee, Hsu, and Lee ${ }^{[29]}$ that investigated the HRQoL of schizophrenic patients from a recovery home, no differences were found between the two genders. This difference in the results can be further investigated in future studies. Compared with patients who were diagnosed with the disease 5 years ago or later (accounting for $9.8 \%$ of the patients), those who were diagnosed with the 
disease 6-10 years ago (accounting for $16.6 \%$ of the patients) reported a higher level of satisfaction for the environmental domain of HRQoL $(F=2.91, p<.05)$. This finding was consistent with that obtained by Shih, ${ }^{[6]}$ who found that there were statistically significant differences in HRQoL between schizophrenic patients with varying disease durations (duration since diagnosis). Furthermore, patients with milder symptom severity reported a higher level of satisfaction for overall HRQoL and the four HRQoL domains. These results were consistent with those of most previous studies. ${ }^{[6,26,30]}$ Compared with the patients who participated less actively in rehabilitation activities, those who took a more active approach reported a higher level of satisfaction for overall HRQoL and the four HRQoL domains. This finding also confirmed Shih's ${ }^{[6]}$ findings, which suggested that $85 \%$ of the patients who participated in community mental health rehabilitation programs experienced an improved quality of life.

\subsection{Schizophrenic patients' empowerment perception and HRQoL}

The results of this study showed that, the higher the empowerment perception, the higher the overall HRQoL and the four HRQoL scores for the schizophrenic patients in the rehabilitation wards. These results were consistent with those obtained by Shih ${ }^{[6]}$ and Corrigan, Faber, Rashid et al. ${ }^{[12]}$ which suggested a significant correlation between the selfdirection of the patients' empowerment perception and their quality of life. Furthermore, Hansson and Björkman ${ }^{[14]}$ and Rogers et al. ${ }^{[19]}$ found that a positive correlation existed between the self-esteem and activism aspects of an individual's empowerment perception and his or her quality of life. Vauth, Kleim, Wirtz et al. ${ }^{[15]}$ and Sibitz, Amering, Unger et al. ${ }^{[20]}$ found that the lack of empowerment perception among schizophrenic patients was linked to their low quality of life. In summary, through their participation in the mental health rehabilitation process and the enhancement of their individual empowerment perception, schizophrenic patients can raise their individual quality of life.

\section{Conclusions}

The results of the study showed that: (1) overall empowerment perception was of a moderately strong level (80.65 \pm 21.56) and the community activism and autonomy domain being the highest scoring domain; (2) the mean score for overall HRQoL was $51.43 \pm 15.7$, which was moderately high, and the social relationship domain generated the highest satisfaction score; (3) there was a significant negative correlation between age and the power-powerlessness domain of empowerment perception, and between symptom severity and the righteous anger domain of empowerment perception; (4) compared with female patients, male patients Published by Sciedu Press enjoyed a higher overall HRQoL and environmental domain HRQoL; (5) compared with patients who were diagnosed 5 years ago or later, those who were been diagnosed 6-10 years ago enjoyed a higher level of satisfaction with respect to the environmental domain of HRQoL; (6) patients who had milder symptom severity and participated more actively in rehabilitation activities enjoyed a higher level of satisfaction for overall HRQoL and the four HRQoL domains; and (7) there was a significant positive correlation between empowerment perception and HRQoL.

\section{Limitations}

This study is affected by two limitations. First, the study utilized a cross-sectional design and was therefore only able to observe the feelings of the schizophrenic patients in the rehabilitation wards at the time of the study's implementation. Second, due to time and manpower constraints, the results of the study cannot be extrapolated to other schizophrenic patients in Taiwan who are also housed in rehabilitation wards.

\section{Implications for practice}

With regard to nursing practice, this study showed that schizophrenic patients scored lowest on the power-powerlessness domain of empowerment perception and the psychological domain of HRQoL. As psychiatric patients in a rehabilitation ward are often encouraged to adhere to the rehabilitation programs developed by medical professionals, it is possible that these medical professionals were regularly limiting these patients' opportunities to exercise their individual power during regular activities. It is therefore proposed that mental health nurses allow their patients to make independent choices and control whenever they interact with them. The results of this study showed a positive correlation between the empowerment perception and HRQoL of patients in rehabilitation wards. Therefore, in this paper we suggest the idea of applying patient partnership in combination with recovery values for mental health and quality of life. When mental health nurses are interacting with patients, they could help them to actively take on disease care responsibilities and encourage these patients to transition from a passive adherence to treatments and submission to professional authority to an active attitude toward participation in disease care, in order to increase their empowerment perception.

\section{ACKNOWLEDGEMENTS}

The authors would like to thank the chronic psychiatric patients who contributed to the survey. We also thank Jianan Psychiatric Center for their support.

\section{CONFLICTS OF INTEREST DisClOSURE}

The authors declare they have no conflicts of interest. 


\section{REFERENCES}

[1] Gale J, Marshall-Lucette S. Community mental health nurses' perspectives of recovery-oriented practice: Mental health nurses' recovery-oriented practice. Journal of Psychiatric and Mental Health Nursing. 2012; 19(4): 348-353. PMid: 22070737. https ://doi.or $\mathrm{g} / 10.1111 / \mathrm{j} .1365-2850.2011 .01803 . \mathrm{x}$

[2] Hsiao CY, Lu HL, Tsai YF. Effect of family sense of coherence on internalized stigma and health-related quality of life among individuals with schizophrenia. International Journal of Mental Health Nursing. 2018; 27(1): 138-146. PMid: 28054432. ttps: //doi.org/10.1111/inm.12302

[3] Gladis MM, Gosch EA, Dishuk NM, et al. Quality of life: Expanding the scope of clinical significance. Journal of Consulting and Clinical Psychology. 1999; 67(3): 320-331. PMid: 10369052. https://doi.org/10.1037/0022-006X.67.3.320

[4] Su CT, Yang AL, Lin CY. The construct of the schizophrenia quality of life scale revision 4 for the population of Taiwan. Occupational Therapy International. 2017. PMid: 29097970. https: //doi.org/10.1155/2017/5328101

[5] Tseng CJ, Chiou JY, Yen WJ, et al. Associations between stigma perception and stigma coping behavior and quality of life in schizophrenic patients treated at a community rehabilitation center. The Journal of Nursing. 2012; 59(4): 62-70.

[6] Shih YW. The exploration of empowerment perception and quality of life among chronic schizophrenia in psychiatric community rehabilitation institution. (Unpublished Master's thesis). National Digital Library of Theses and Dissertations in Taiwan; 2009.

[7] Liu HY, Lin ML, Ma WF. A nursing experience of using recovery theory to improve the patient having self-harm behavior. Journal of Psychiatric Mental Health Nursing. 2015; 10(1): 26-33.

[8] World Health Organization. Schizophrenia. 2014. Available from: http://www. who.int/topics/schizophrenia/en/

[9] Lee MC, Hsiung DY, Lin EC. Discharge planning service for a chronic schizophrenia patient. Yuan-Yuan Nursing. 2010; 4(1): 6877.

[10] Wu LM, Hong CH, Chen CH. Application of empowerment in the organization. The Journal of Nursing. 2006; 53(6): 53-58.

[11] Rogers ES, Chamberlin J, Ellison ML, et al. A consumer-constructed scale to measure empowerment among users of mental health services. Psychiatric Services. 1997; 48(8): 1042-1047. PMid: 9255837. https://doi.org/10.1176/ps.48.8.1042

[12] Corrigan PW, Faber D, Rashid F, et al. The construct validity of empowerment among consumers of mental health services. Schizophrenia Research. 1999; 38(1): 77-84. https://doi.org/10.1016/ S0920-9964(98) 00180-7

[13] Jana AK, Ram D, Praharaj SK. Empowerment and its associations in schizophrenia: a cross-sectional study. Community Mental Health Journal. 2014; 50(6): 697-701. PMid: 24794840. https: //doi.org/10.1007/s10597-014-9729-4

[14] Hansson L, Björkman T. Empowerment in people with a mental illness: Reliability and validity of the Swedish version of an empowerment scale. Scandinavian Journal of Caring Sciences. 2005; 19(1): 32-38. PMid: 15737163. https://doi .org/10.1111/j.1471-6 $712.2004 .00310 . x$

[15] Vauth R, Kleim B, Wirtz M, et al. Self-efficacy and empowerment as outcomes of self-stigmatizing and coping in schizophre- nia. Psychiatry Research. 2007; 150(1): 71-80. PMid: 17270279. https://doi.org/10.1016/j.psychres.2006.07.005

[16] Lee SC, Yu WH, Chiu EC, et al. Minimal detectable change of the quality of life for mental disorder scale in patients with schizophrenia. Journal of Occupational Therapy Association. 2015; 33(2): 132-148.

[17] Tseng SH. A related factor analysis of self-awareness on quality of life among schizophrenic patients in the community (Unpublished Master's thesis). National Digital Library of Theses and Dissertations in Taiwan; 2006.

[18] Chou KR, Shih YW, Chang C, et al. Psychosocial rehabilitation activities, empowerment, and quality of community-based life for people with schizophrenia. Archives of Psychiatric Nursing. 2012; 26(4): 285-294. PMid: 22835748. https : //doi.org/10.1016/j . apnu .2012 .04 .003

[19] Rogers ES, Ralph RO, Salzer MS. Validating the empowerment scale with a multisite sample of consumers of mental health services. Psychiatric Services. 2010; 61(9): 933-936. PMid: 20810594. https://doi.org/10.1176/ps.2010.61.9.933

[20] Sibitz I, Amering M, Unger A, et al. The impact of the social network, stigma and empowerment on the quality of life in patients with schizophrenia. European Psychiatry. 2011; 26(1): 28-33. PMid: 21036554. https://doi.org/10.1016/j.eurpsy.2010.08.0 10

[21] Hang YH, Rong JR, Lin SK, et al. The mediating effect of community living skills on stigma perception and quality of life for the patients with schizophrenia. The Journal of Psychiatric Mental Health Nursing. 2015; 10(1): 1-8.

[22] Chang TJ, Hwu HG, Wei FW. Interpersonal reliability of the BPRS brief psychiatric rating scale (BPRS). Chinese Society of the Neurology and Psychiatry Bulletin. 1986; 12(1): 29-36.

[23] Chen SC, Liu CY, Hsieh CJ, et al. Psychometric testing of a Chinese Empowerment Scale for the patients with chronic mental illness. The Journal of Psychiatric Mental Health Nursing. 2017; 12(1): 5-13.

[24] Yao KP. Introduction to the development of the WHOQOL-Taiwan Version, Chinese Journal of Public Health. 2000; 19(4): 315-324.

[25] Yao KP. Taiwan version of the World Health Organization Quality of Life Questionnaire Development and Use Manual (2nd ed). Taipei: Taiwan version of the World Health Organization Quality of Life Questionnaire Development; 2005.

[26] Guo TZ. Factors associated with the quality of life among rehabilitation inpatients with chronic mental illness (Unpublished Master's thesis). National Digital Library of Theses and Dissertations in Taiwan; 2009.

[27] Huang YM, Kao CY, Hsu JH, et al. Analyses of the quality of life among patients with schizophrenia. Taiwanese Journal of Psychiatry. 2011; 25(3): 158-166

[28] Li IL, Shiau SJ, Li SH, et al. The construction of essential competence of community psychiatric mental health nursing-self construction. The Journal of Psychiatric Mental Health Nursing. 2008; 3(2): 10-18

[29] Lee CH, Hsu HY, Lee YC. Demographic influences on quality of life among patients with schizophrenia in halfway house. Journal of Mei Ho University. 2013; 32(1): 27-40.

[30] Kao CC, Huang HM. A comparison of the quality of life of patients with schizophrenia in daycare and homecare settings. The Journal of Nursing Research. 2014; 22(2): 126-135. PMid: 24821420. https ://doi.org/10.1097/jnr.0000000000000026 